heterosexual males. Our findings imply that FPL are not useful in identifying HPV infections with a high transmission potential in this population.

Disclosure No significant relationships.

\section{P521 INCREASES IN THE ESTIMATED NUMBER OF REPORTED GONORRHEA CASES AMONG MEN WHO HAVE SEX WITH MEN (MSM): THE ROLE OF TESTING}

${ }^{1}$ Austin Williams, ${ }^{1}$ Emily Weston*, ${ }^{2}$ Thomas Gift, ${ }^{1}$ Elizabeth Torrone. ${ }^{1}$ US Centers for Disease Control and Prevention, Division of STD Prevention, Atlanta, USA; ${ }^{2}$ Centers for Disease Control and Prevention, Division of STD Prevention, Atlanta, USA

\subsection{6/sextrans-2019-sti.599}

Background Interpreting trends in rates of reported cases of gonorrhea is difficult because infections, particularly extra-genital infections, are often asymptomatic and increased screening coverage will result in increased case detection. In the absence of national data on screening coverage among men who have sex with men (MSM), we estimated trends in MSM testing in the United States given a range of positivity estimates.

Methods We estimated the number of tests that would have had to occur to identify the estimated number of reported gonorrhea cases among MSM in a given year. The number of tests was calculated by dividing the annual number of estimated cases among MSM (based on sentinel surveillance) by estimated test positivity among MSM (based on published literature). We calculated the estimated number of tests under both low positivity (3.5\%) and high positivity (10\%), as well as the effect of stable and changing positivity during 20162017.

Results The estimated number of reported gonorrhea cases among MSM increased by 17.9\% during 2016-2017 (from 163,537 to 192,740 ). We estimated that 1.9 million (assuming $10 \%$ positivity) to 5.5 million (assuming $3.5 \%$ positivity) tests performed among MSM would be needed to detect the estimated number of gonorrhea cases among MSM in 2017. This represents an increase of approximately 290,000 to 830,000 tests over the estimated number of tests conducted in 2016. Alternatively, if the number of tests was stable over time, a 0.6 to 1.8 percentage point increase in positivity from 2016 to 2017 would be consistent with the increase in observed cases.

Conclusion We provide a framework to inform trends in case rates by analyzing the impact of changes in positivity and testing over time. Our analysis implies that estimated increases in reported gonorrhea cases among MSM likely resulted from both increased screening and increased incidence.

Disclosure No significant relationships.

\section{P522 DISPARITIES IN HIVISTI TESTING AND DIAGNOSIS AMONG URBAN AND NON-URBAN US MEN WHO HAVE SEX WITH MEN FROM 2013 TO 2017}

Maria Zlotorzynska*, Travis Sanchez, Patrick Sullivan. Emory University Rollins School of Public Health, Department of Epidemiology, Atlanta, USA

\subsection{6/sextrans-2019-sti.600}

Background Most data about HIV/STI testing and diagnosis among US men who have sex with men (MSM) comes from urban areas, though the majority of the population resides outside of these areas. Since 2013, the American Men's Internet Survey (AMIS) has conducted annual nationwide online behavioral surveillance of $\geq 10,000$ US MSM.

Methods Participants age $15+$ were recruited through online advertisements. County urban/rural categories were based on National Center for Health Statistics classification. Poisson models using generalized estimating equations tested associations between urban/rural category and HIV testing, STI (syphilis, gonorrhea, chlamydia) testing and STI diagnoses in the past 12 months. All models controlled for survey year, age, race/ethnicity, insurance, HIV status (except model for HIV testing) and recruitment source.

Results From 2013 through 2017, 49,903 completed surveys were collected: $42.4 \%$ MSM from urban counties, $20.5 \%$ suburban, $28.3 \%$ small/medium metro, and $8.8 \%$ rural. STI testing was more prevalent in urban counties (50.2\%) compared to suburban $(37.8 \%, p<0.0001)$, small/medium metro $(35.6 \%, p<0.0001)$ and rural $(27.8 \%, p<0.0001)$ counties. STI diagnoses were more prevalent in urban counties (13.4\%) compared to suburban $(8.1 \%, p<0.0001)$, small/ medium metro $7.5 \%, p<0.0001)$ and rural $(5.4 \%$, $p<0.0001)$ counties. Among HIV-negative/unknown status MSM, HIV testing was more prevalent among MSM from urban counties (61.9\%) compared to suburban $(52.3 \%$, $p<0.0001)$, small/medium metro $(50.6 \%, p<0.0001)$ and rural $(43.6 \%, p<0.0001)$ counties. Significant trends over time were observed in HIV testing for all counties, while STI testing only increased in urban and small/medium metro counties. STI diagnoses increased significantly in all but rural counties.

Conclusion Urban/rural disparities in HIV/STI testing and STI diagnoses were found in a multi-year national sample of US MSM. These findings likely reflect disparate geographical distribution of healthcare access and resources. If these disparities cannot be adequately addressed in programs that reach underserved areas, nationwide HIV/STI prevention goals for MSM will not likely be met.

Disclosure No significant relationships.

\section{P523 URETHRAL MICROBIOTA IN IDIOPATHIC NON- GONOCOCCAL URETHRITIS (NGU) IN MEN WHO HAVE SEX WITH MEN AND MEN WHO HAVE SEX WITH WOMEN}

${ }^{1}$ Sujatha Srinivasan*, ${ }^{2}$ Laura Chambers, ${ }^{3}$ Ken Tapia, ${ }^{4}$ Noah Hoffman, ${ }^{1}$ Matthew Munch, ${ }^{5}$ Jennifer Morgan, ${ }^{1}$ Daniel Domogala, ${ }^{6} \mathrm{M}$ Lowens, ${ }^{4}$ Sean Proll, ${ }^{7} \mathrm{M}$ Huang, ${ }^{1}$ Keith Jerome, ${ }^{4}$ Matthew Golden, ${ }^{8}$ James Hughes, 'David Fredricks, ${ }^{2}$ Lisa Manhart. ${ }^{1}$ Fred Hutchinson Cancer Research Center, Vaccine and Infectious Disease Division, Seattle, USA; ${ }^{2}$ University of Washington, Epidemiology, Seattle, USA; ${ }^{3}$ University of Washington, Global Health, Seattle, USA; ${ }^{4}$ University of Washington, Medicine, Seattle, USA; ${ }^{5}$ Public Health - Seattle and King County, Seattle, USA; ${ }^{6}$ Public Health - Seattle and King County, HIVISTD Program, Seattle, USA; ' University of Washington, Laboratory Medicine, Seattle, USA; ${ }^{8}$ University of Washington, Biostatistics, Seattle, USA

10.1136/sextrans-2019-sti.601

Background NGU is common with no known etiology in $\sim 50 \%$ of cases. We evaluated the association of urethral bacteria with NGU among men who have sex with men (MSM) and men who have sex with women (MSW).

Methods Urine samples were collected from MSM and MSW attending a Seattle STD Clinic and enrolled in a cross-sectional study. Chlamydia trachomatis (CT) and Mycoplasma genitalium (MG) were detected by TMA (Aptima), and adenovirus, HSV-1 and HSV-2 by PCR. NGU was defined as having urethral 